\title{
Supplementary Data Set
}

Text

Tables 1-4

References

\section{Criteria used to infer primary mineralogy}

The following criteria are used to infer the primary mineralogy of fossilized skeletons (shells, tests, sclerites, spicules, teeth, etc.) and abiotic precipitates (ooids, marine synsedimentary cements):

(1) Detection of original element concentrations either from fluid inclusions in precipitates or from skeletal material (magnesium - for low- or high-Mg calcite; strontium - for aragonite).

(2) Preservation of specific skeletal fabrics either in calcite or in phosphate minerals' and silica replicas (e.g., foliated and prismatic microstructure - low $\mathrm{Mg}$-calcite; microgranular microstructure - high-Mg calcite; nacreous and lamello-fibrillar microstructures - aragonite).

(3) Relative quality of preservation of different precipitates in the same sample: fabric preserved - low-Mg calcite; fabric preserved and spar-filled molds with microdolomite - high-Mg calcite; coarse spar mosaic-filled molds, generally irregularly cross-cutting original structure - aragonite.

(4) Epitaxial synsedimentary marine cements developing in optical continuity with skeletal elements (skeletons only): bladed equant calcite - low-Mg calcite; fibrous calcite - high Mg-calcite; botryoids of acicular crystals - aragonite.

(5) Phylogenetic application of skeletal mineralogies in extant groups to their probable fossil relatives.

(6) Relative stable isotope composition $\left(\delta^{13} \mathrm{C}, \delta^{18} \mathrm{O}\right)$ of different precipitates in the same sample with less altered signatures characterizing low-Mg calcite fabrics.

Table 1. Inferred primary mineralogy of taxa listed on Figure 1 (Distribution of major skeletal taxa and carbonate abiotic precipitates (ooids and synsedimentary marine cements) from the Upper Ediacaran to Middle Ordovician). FAD - first appearance datum: E - Upper Ediacaran, IND - lower Nemakit-Daldynian, uND - upper NemakitDaldynian, IT - lower Tommotian, $\mathrm{mT}$ - middle Tommotian, uT - upper Tommotian, 1A - lower Atdabanian, uA - upper Atdabanian, B - Botoman, MC - Middle Cambrian (Cambrian Series 3), UC - Upper Cambrian (Furongian), LO - Lower Ordovician, MO - Middle Ordovician; A - aragonite, HMC - high Mg-calcite, LMC - low Mg-calcite, P - phosphate.

\begin{tabular}{|l|l|l|l|l|}
\hline Taxon & FAD & $\begin{array}{l}\text { Inferred } \\
\text { mineralogy }\end{array}$ & Criteria & Comments and references \\
\hline Cloudina-group & E & HMC & $2-4$ & $(S 1-S 3)$ \\
\hline Namapoikia & E & A & 3 & $(S 4)$ \\
\hline Namacalathus-gr. & E & HMC & $2-4$ & $(S 3, S 5)$ \\
\hline Sinotubilites & E & A & 2 & $(S 6)$ \\
\hline Chaetognatha & IND & P & 2,3 & $\begin{array}{l}\text { including protoconodonts }(S 7, \\
\text { S8); FAD }(S 9)\end{array}$ \\
\hline Anabaritida & IND & A & 2,3 & $(S 10, S 11) ;$ FAD $(S 9)$ \\
\hline Orthothecimorpha & UND & A & 2,3 & $(S 12, S 13) ;$ FAD $(S 9)$ \\
\hline
\end{tabular}




\begin{tabular}{|c|c|c|c|c|}
\hline Helcionelliformes & uND & A & 2,3 & $(S 14-S 18) ;$ FAD (S9) \\
\hline Paragastropoda & uND & A & 2,3 & $(S 14-S 17) ;$ FAD $(S 9)^{-}$ \\
\hline Coelosclerotophora & uND & $\mathrm{A}$ & 2,3 & $\begin{array}{l}\text { including chancelloriids (S16- } \\
S 20) ; \text { FAD }(S 9)\end{array}$ \\
\hline Renalcida & IND & HMC & 2,3 & $(S 21)$, herein; FAD $(S 22)$ \\
\hline Cambroclavida & 1T & A & & $(S 16, S 23) ; \mathrm{FAD}(S 24)$ \\
\hline Tommotiida & 1T & $\mathrm{P}$ & 2,3 & $(S 25) ; \mathrm{FAD}(S 9)$ \\
\hline Linguliformea & $1 \mathrm{~T}$ & $\mathrm{P}$ & 2,5 & $(S 26, S 27) ; \mathrm{FAD}(S 9)$ \\
\hline Hyolithelmintida & 1T & $\mathrm{P}$ & 2,3 & $(S 28, S 29) ; \mathrm{FAD}(S 9)$ \\
\hline Paracarinachitidae & 1T & $\mathrm{A}$ & 2,3 & $(S 30)$ \\
\hline Conulariida & IT & $\mathrm{P}$ & 2,3 & $\begin{array}{l}\text { including hexangulaconulariids } \\
(S 31, S 32)\end{array}$ \\
\hline Rostroconchia & IT & $\mathrm{A}$ & 2,3 & $(S 17, S 33, S 34) ;$ FAD $(S 9)$ \\
\hline Bivalvia & 1T & $\mathrm{A}$ & 2,3 & $(S 14, S 35-S 37)$ \\
\hline Archaeocyatha & IT & HMC & $1-6$ & $\begin{array}{l}(S 21, S 38, S 39), \text { herein; FAD } \\
(S 40)\end{array}$ \\
\hline Cribricyatha & $\mathrm{mT}$ & HMC & 2,3 & herein; FAD $(S 40)$ \\
\hline Hyolithomorpha & $1 \mathrm{~T}$ & $?$ & & FAD $(S 40)$ \\
\hline Coleoloida & $1 \mathrm{~T}$ & $\mathrm{~A}$ & 2,3 & $(S 41) ; \mathrm{FAD}(S 40)$ \\
\hline Obolellata & $\mathrm{mT}$ & HMC & $2,3,5$ & $(S 42, S 43)$ \\
\hline Calcarea & $\mathrm{mT}$ & HMC & $2,3,5$ & $(S 21, S 44) ;$ FAD $(S 39)$ \\
\hline Khasaktiidae & $\mathrm{mT}$ & HMC & $1-4,6$ & $(S 38, S 45)$ \\
\hline Radiocyatha & $\mathrm{mT}$ & $\mathrm{A}$ & 3,4 & $(S 46) ; \mathrm{FAD}(S 40)$ \\
\hline Tabulaconida & B & $\mathrm{A}$ & $1-4,6$ & $\begin{array}{l}\text { modular species only }(S 44, S 47 \text {, } \\
S 48)\end{array}$ \\
\hline Mobergellidae & $\mathrm{uT}$ & $\mathrm{P}$ & 2,3 & $(S 49) ;$ FAD $(S 40)$ \\
\hline Hydroconozoa & $\mathrm{uT}$ & LMC & $1-4,6$ & $(S 38, S 39, S 50)$ \\
\hline Stenothecoida & $1 \mathrm{~A}$ & LMC & 2,3 & $(S 42) ; \mathrm{FAD}(S 40)$ \\
\hline Trilobita & $1 \mathrm{~A}$ & LMC & $1-4,6$ & $(S 21, S 51-S 54) ; \mathrm{FAD}(S 40)$ \\
\hline Agmata & $\mathrm{B}$ & LMC & 2,3 & $(S 21) ; \mathrm{FAD}(S 55)$ \\
\hline Ostracoda & $\mathrm{LO}$ & LMC & $2,3,5$ & $(S 56, S 57) ;$ FAD $(S 58)$ \\
\hline Rhynchonellata & $1 \mathrm{~A}$ & LMC & $\begin{array}{l}2,3,5 \\
6\end{array}$ & $(S 42, S 59)$ \\
\hline Kutorginata & $\mathrm{uT}$ & LMC & 2,3 & not shown on Fig. 1 (S59) \\
\hline Echinodermata & $\mathrm{uA}$ & HMC & $1-5$ & $(S 21, S 60) ; \mathrm{FAD}(S 40)$ \\
\hline Palaeoscolecida & $\mathrm{uA}$ & $\mathrm{P}$ & 2,3 & (S61-S63); FAD (S40) \\
\hline Tannuolinidae & $\mathrm{uA}$ & $\mathrm{P}$ & 2,3 & $(S 64) ;$ FAD $(S 24)$ \\
\hline Byroniida & $\mathrm{uA}$ & $\mathrm{P}$ & 2,3 & $(S 65) ; \mathrm{FAD}(S 16)$ \\
\hline Tardypolipoda & $\mathrm{uA}$ & $\mathrm{P}$ & 2,3 & $\begin{array}{l}\text { Microdyction and other Cambrian } \\
\text { sclerite-bearing lobopodians } \\
(\text { S66); FAD }(S 40)\end{array}$ \\
\hline Conodonta & $\mathrm{MC}$ & $\mathrm{P}$ & 2,5 & $(S 7)$ \\
\hline Bryozoa & $\mathrm{LO}$ & LMC & $2,3,5$ & (S67-S69); FAD (S70) \\
\hline Stromatoporata & MO & LMC & 2,3 & $(S 71) ;$ FAD $(S 72)$ \\
\hline Tabulata & $\mathrm{LO}$ & LMC & 2,3 & $(S 73) ; \mathrm{FAD}(S 74)$ \\
\hline Rugosa & $\mathrm{MO}$ & LMC & 2,3 & $(S 73) ; \mathrm{FAD}(S 74)$ \\
\hline Cephalopoda & $\mathrm{UC}$ & HMC? & 5 & $(S 75-S 77)$ \\
\hline Polyplacophora & $\mathrm{UC}$ & HMC? & 5 & $(S 78, S 79) ; \mathrm{FAD}(S 80)$ \\
\hline
\end{tabular}


Table 2. Inferred primary mineralogy of ooids indicated on Figure 1 (Distribution of major skeletal taxa and carbonate abiotic precipitates (ooids and synsedimentary marine cements) from the Upper Ediacaran to Middle Ordovician). E - Ediacaran, ND -

Nemakit-Daldynian, T -Tommotian, 1A - lower Atdabanian, uA - upper Atdabanian, B

- Botoman, Tn - Toyonian; A - aragonite, HMC - high Mg-calcite, LMC - low Mgcalcite.

\begin{tabular}{|c|c|c|c|c|}
\hline $\begin{array}{l}\text { Formation; locality; } \\
\text { paleocontinent }\end{array}$ & Age & $\begin{array}{l}\text { Inferred } \\
\text { mineralogy }\end{array}$ & Criteria & $\begin{array}{l}\text { Comments and } \\
\text { references }\end{array}$ \\
\hline $\begin{array}{l}\text { Biri Formation; Norway; } \\
\text { Baltica }\end{array}$ & $\mathrm{E}$ & A & 3 & $(S 81)$ \\
\hline $\begin{array}{l}\text { Islay Limestone, } \\
\text { Kingston Peak } \\
\text { Formation; Scotland, } \\
\text { UK; Avalonia }\end{array}$ & $\mathrm{E}$ & $\mathrm{A}$ & 3 & $(S 82)$ \\
\hline $\begin{array}{l}\text { Virgin Spring } \\
\text { Limestone; California, } \\
\text { USA; Laurentia }\end{array}$ & $\mathrm{E}$ & $\mathrm{A}$ & 3 & $(S 83)$ \\
\hline $\begin{array}{l}\text { Trezona Formation; } \\
\text { South Australia; Eastern } \\
\text { Gondwana }\end{array}$ & $\mathrm{E}$ & $\mathrm{A}$ & 3 & $(S 84)$ \\
\hline $\begin{array}{l}\text { Wonoka Formation; } \\
\text { South Australia; Eastern } \\
\text { Gondwana }\end{array}$ & $\mathrm{E}$ & $\mathrm{A}$ & 3 & $(S 82)$ \\
\hline $\begin{array}{l}\text { Série Lie de Vin; Anti- } \\
\text { Atlas, Morocco; Western } \\
\text { Gondwana }\end{array}$ & $\mathrm{T}$ & $\mathrm{A}, \mathrm{HMC}$ & 3 & $(S 82)$; age $(S 85)$ \\
\hline $\begin{array}{l}\text { Parachilna Formation; } \\
\text { South Australia; Eastern } \\
\text { Gondwana }\end{array}$ & $\mathrm{T}$ & A, HMC & 3 & $(S 82)$ \\
\hline $\begin{array}{l}\text { Lower Wilkawillina } \\
\text { Limestone; South } \\
\text { Australia; Eastern } \\
\text { Gondwana }\end{array}$ & $1 \mathrm{~A}$ & LMC & 3 & $(S 82)$ \\
\hline $\begin{array}{l}\text { lower Churan Member, } \\
\text { Pestrotsvet Formation; } \\
\text { southern Yakutia, } \\
\text { Russia; Siberia }\end{array}$ & $1 \mathrm{~A}$ & LMC & 3 & $(S 86)$ \\
\hline $\begin{array}{l}\text { upper Churan Member, } \\
\text { Pestrotsvet Formation; } \\
\text { southern Yakutia, } \\
\text { Russia; Siberia }\end{array}$ & $\mathrm{uA}$ & $\mathrm{HMC}$ & 3 & $(S 86)$ \\
\hline $\begin{array}{l}\text { Salaany Gol Formation; } \\
\text { western Mongolia; } \\
\text { Zavkhan terrane }\end{array}$ & A & A, HMC & 3 & $(S 46)$ \\
\hline $\begin{array}{l}\text { Amouslek Formation; } \\
\text { Anti-Atlas, Morocco; } \\
\text { Western Gondwana }\end{array}$ & $\mathrm{uA}$ & A & 3 & $(S 82)$; age $(S 87)$ \\
\hline $\begin{array}{l}\text { upper Wood Canyon } \\
\text { Formation; California, } \\
\text { USA; Laurentia }\end{array}$ & $\begin{array}{l}\mathrm{uA-} \\
\mathrm{B}\end{array}$ & HMC/A(?) & 3 & $(S 88)$ \\
\hline
\end{tabular}




\begin{tabular}{|c|c|c|c|c|}
\hline $\begin{array}{l}\text { unnamed formation; } \\
\text { Ellsworth Mountains, } \\
\text { West Antarctica; Eastern } \\
\text { Gondwana }\end{array}$ & B & A & 3 & $(S 89) ;$ age $(S 90)$ \\
\hline $\begin{array}{l}\text { erratics; South Shetland } \\
\text { Islands, Antarctica; } \\
\text { Eastern Gondwana }\end{array}$ & B & A & 3 & $(S 90)$ \\
\hline $\begin{array}{l}\text { Punta Manna Member; } \\
\text { Nebida Formation; } \\
\text { Sardinia, Italy; Western } \\
\text { Gondwana }\end{array}$ & B & A & 3 & $(S 91)$ \\
\hline $\begin{array}{l}\text { Mural Limestone; British } \\
\text { Columbia, Canada; } \\
\text { Laurentia }\end{array}$ & $\mathrm{B}$ & HMC & 3 & $(S 92) ;$ age $(S 93)$ \\
\hline $\begin{array}{l}\text { Forteau Formation; } \\
\text { Labrador-Newfoundland, } \\
\text { Canada; Laurentia }\end{array}$ & B & A, HMC & 3 & $(S 21) ;$ age $(S 93)$ \\
\hline $\begin{array}{l}\text { Shady Dolomite; } \\
\text { Virginia, USA; Laurentia }\end{array}$ & B & $\mathrm{HMC}$ & 3 & $(S 94) ;$ age $(S 95)$ \\
\hline $\begin{array}{l}\text { Lastours Formation; } \\
\text { Montagne Noire, France; } \\
\text { Western Gondwana }\end{array}$ & B & $\mathrm{HMC}$ & 3 & $(S 96)$ \\
\hline $\begin{array}{l}\text { Burj Formation; Jordan; } \\
\text { Western Gondwana }\end{array}$ & $\mathrm{Tn}$ & $\mathrm{HMC}$ & 3 & $(S 97)$ \\
\hline $\begin{array}{l}\text { Ledger Formation; } \\
\text { Pennsylvania, USA; } \\
\text { Laurentia }\end{array}$ & Tn? & $\mathrm{HMC}$ & 3 & $(S 98)$ \\
\hline $\begin{array}{l}\text { Port au Port Group; } \\
\text { Newfoundland, Canada; } \\
\text { Laurentia }\end{array}$ & $\begin{array}{l}\text { MC- } \\
\text { UC }\end{array}$ & $\begin{array}{l}\text { HMC, A, } \\
\text { LMC }\end{array}$ & 3,4 & $\begin{array}{l}\text { LMC ooids were not } \\
\text { indicated in }(S 99) \text { but } \\
\text { some of them show optical } \\
\text { continuity with nuclei of } \\
\text { trilobite fragments }\end{array}$ \\
\hline
\end{tabular}

Table 3. Inferred primary mineralogy of synsedimentary marine cements indicated on Figure 1 (Distribution of major skeletal taxa and carbonate abiotic precipitates (ooids and synsedimentary marine cements) from the Upper Ediacaran to Middle Ordovician). E - Ediacaran, ND -Nemakit-Daldynian, T - Tommotian, A - Atdabanian, uA - upper Atdabanian, B - Botoman, MC - Middle Cambrian (Cambrian Series 3), UC - Upper Cambrian (Furongian), UO - Upper Ordovician; A - aragonite, HMC - high Mgcalcite, LMC - low Mg-calcite.

\begin{tabular}{|l|l|l|l|l|}
\hline $\begin{array}{l}\text { Formation; locality; } \\
\text { paleocontinent }\end{array}$ & Age & $\begin{array}{l}\text { Inferred } \\
\text { mineralogy }\end{array}$ & Criteria & $\begin{array}{l}\text { Comments and } \\
\text { references }\end{array}$ \\
\hline $\begin{array}{l}\text { Biri Formation; Norway; } \\
\text { Baltica }\end{array}$ & E & A & 3 & $(S 81)$ \\
\hline $\begin{array}{l}\text { Islay Limestone, Kingston } \\
\text { Peak Formation; Scotland, } \\
\text { UK; Avalonia }\end{array}$ & E & A & 3 & $(S 82)$ \\
\hline $\begin{array}{l}\text { Virgin Spring Limestone; } \\
\text { California, USA; Laurentia }\end{array}$ & E & A & 3 & $(S 83)$ \\
\hline Trezona Formation; South & E & A & 3 & $(S 84)$ \\
\hline
\end{tabular}




\begin{tabular}{|c|c|c|c|c|}
\hline Australia; Eastern Gondwana & & & & \\
\hline $\begin{array}{l}\text { Wonoka Formation; South } \\
\text { Australia; Eastern Gondwana }\end{array}$ & $\mathrm{E}$ & $\mathrm{A}$ & 3 & $(S 82)$ \\
\hline $\begin{array}{l}\text { Série Lie de Vin; Anti-Atlas, } \\
\text { Morocco; Western Gondwana }\end{array}$ & $\mathrm{T}$ & A & 3 & $(S 82) ;$ age $(S 85)$ \\
\hline $\begin{array}{l}\text { Parachilna Formation; South } \\
\text { Australia; Eastern Gondwana }\end{array}$ & $\mathrm{T}$ & A & 3 & $(S 82)$ \\
\hline $\begin{array}{l}\text { Isit' Member, Pestrotsvet } \\
\text { Formation; southern Yakutia, } \\
\text { Russia; Siberia }\end{array}$ & $\mathrm{T}$ & HMC & 3 & $(S 39)$ \\
\hline $\begin{array}{l}\text { Negyurchene Biohermal and } \\
\text { Oy-Muran Reef massives; } \\
\text { southern Yakutia, Russia; } \\
\text { Siberia }\end{array}$ & A & A & 3 & $\begin{array}{l}\text { aragonite botryoids are } \\
\text { cited as calcified alga } \\
\text { Zaganolomia in }(S 100)\end{array}$ \\
\hline $\begin{array}{l}\text { Salaany Gol Formation; } \\
\text { western Mongolia; Zavkhan } \\
\text { terrane }\end{array}$ & A & A, HMC & 3 & $(S 46)$ \\
\hline $\begin{array}{l}\text { Amouslek Formation; Anti- } \\
\text { Atlas, Morocco; Western } \\
\text { Gondwana }\end{array}$ & $\mathrm{uA}$ & A & 3 & $(S 82) ;$ age $(S 87)$ \\
\hline $\begin{array}{l}\text { Upper Wilkawillina } \\
\text { Limestone; South Australia; } \\
\text { Eastern Gondwana }\end{array}$ & $\mathrm{uA}$ & $\mathrm{A}, \mathrm{HMC}$ & 1,3 & $(S 101)$ \\
\hline $\begin{array}{l}\text { Mural Limestone; British } \\
\text { Columbia, Canada; Laurentia }\end{array}$ & B & A, HMC & 3 & $(S 102) ;$ age $(S 93)$ \\
\hline $\begin{array}{l}\text { Sekwi Formation; Yukon } \\
\text { Territory, Canada; Laurentia }\end{array}$ & B & $\mathrm{A}, \mathrm{HMC}$ & 3 & $(S 103) ;$ age $(S 93)$ \\
\hline $\begin{array}{l}\text { Forteau Formation; Labrador- } \\
\text { Newfoundland, Canada; } \\
\text { Laurentia }\end{array}$ & B & A, HMC & 3 & $(S 21) ;$ age $(S 95)$ \\
\hline $\begin{array}{l}\text { March Point Formation; } \\
\text { Newfoundland, Canada; } \\
\text { Laurentia }\end{array}$ & $\mathrm{MC}$ & A & 3 & $(S 104)$ \\
\hline $\begin{array}{l}\text { Mila Formation; Iran; } \\
\text { Western Gondwana }\end{array}$ & UC & LMC & 3 & $(S 105)$ \\
\hline $\begin{array}{l}\text { Wilberns Formation; Texas, } \\
\text { USA; Laurentia }\end{array}$ & $\mathrm{UC}$ & LMC & 1,3 & $(S 106)$ \\
\hline $\begin{array}{l}\text { Effna Formation; Virginia, } \\
\text { USA; Laurentia }\end{array}$ & $\mathrm{UO}$ & LMC & 3,6 & $(S 107)$ \\
\hline
\end{tabular}

Table 4. Stratigraphic distribution and location of carbonate flat-pebble conglomerates in Cambrian and Lower Ordovician. B - Botoman, Tn - Toyonian, IMC - lower Middle Cambrian (Cambrian Series 3), uMC - upper Middle Cambrian (Cambrian Series 3), UC - Upper Cambrian (Furongian), LO - Lower Ordovician.

\begin{tabular}{|l|l|l|}
\hline Formation; locality; paleocontinent & Age & $\begin{array}{l}\text { Comments and } \\
\text { references }\end{array}$ \\
\hline $\begin{array}{l}\text { Sellick Hill Formation; South Australia; Eastern } \\
\text { Gondwana }\end{array}$ & B & (S108); age (S109) \\
\hline Agaleva Formation; Krasnoyarsk Region, Russia; & B & cited as calcareous \\
\hline
\end{tabular}




\begin{tabular}{|c|c|c|}
\hline Siberia & & breccia in $(S 110)$ \\
\hline Barylay Formation; Yakutia, Russia; Siberia & Tn & AZ, pers. Observation \\
\hline $\begin{array}{l}\text { Zaledeevo Formation; Krasnoyarsk Region, Russia; } \\
\text { Siberia }\end{array}$ & IMC & $\begin{array}{l}\text { cited as calcareous } \\
\text { breccia in }(S 110)\end{array}$ \\
\hline $\begin{array}{l}\text { Litvintsevo Formation; Krasnoyarsk Region, } \\
\text { Russia; Siberia }\end{array}$ & IMC & $\begin{array}{l}\text { cited as calcareous } \\
\text { breccia in }(S 110)\end{array}$ \\
\hline Dereskir Formation; Yakutia, Russia; Siberia & IMC & $\begin{array}{l}\text { cited as calcareous } \\
\text { breccia in }(S 110)\end{array}$ \\
\hline Elanskoe Formation; Yakutia, Russia; Siberia & IMC & $\begin{array}{l}\text { cited as calcareous } \\
\text { breccia in }(S 110)\end{array}$ \\
\hline $\begin{array}{l}\text { Zonda Formation; Argentine Precordillera; } \\
\text { Occidentalia terrane }\end{array}$ & $\mathrm{MC}$ & $(S 111)$ \\
\hline $\begin{array}{l}\text { La Laja Formation; Argentine Precordillera; } \\
\text { Occidentalia terrane }\end{array}$ & $\mathrm{MC}$ & $(S 112)$ \\
\hline $\begin{array}{l}\text { Jubilee Formation; British Columbia, Canada; } \\
\text { Laurentia }\end{array}$ & $\mathrm{MC}$ & $(S 113)$ \\
\hline upper Wheeller Formation; Utah, USA; Laurentia & $\mathrm{MC}$ & $(S 114)$ \\
\hline Marjum Formation; Utah, USA; Laurentia & $\mathrm{MC}$ & $(S 114)$ \\
\hline Tangha Formation; Yakutia, Russia; Siberia & $\mathrm{uMC}$ & $\begin{array}{l}\text { cited as calcareous } \\
\text { breccia in }(S 110)\end{array}$ \\
\hline Ust'botoma Formation; Yakutia, Russia; Siberia & $\mathrm{uMC}$ & $\begin{array}{l}\text { cited as calcareous } \\
\text { breccia in }(S 110)\end{array}$ \\
\hline $\begin{array}{l}\text { Ust'brus Formation; Krasnoyarsk Region, Russia; } \\
\text { Siberia }\end{array}$ & $\mathrm{uMC}$ & $\begin{array}{l}\text { cited as calcareous } \\
\text { breccia in }(S 110)\end{array}$ \\
\hline $\begin{array}{l}\text { Labazny Formation; Krasnoyarsk Region, Russia; } \\
\text { Siberia }\end{array}$ & $\mathrm{uMC}$ & $\begin{array}{l}\text { cited as calcareous } \\
\text { breccia in }(S 110)\end{array}$ \\
\hline North China; Eastern Gondwana & $\begin{array}{l}\text { MC- } \\
\mathrm{UC}\end{array}$ & $(S 115)$ \\
\hline Mila Formation; Iran; Western Gondwana & $\mathrm{UC}$ & $(S 105)$ \\
\hline $\begin{array}{l}\text { Port au Port Group; Newfoundland, Canada; } \\
\text { Laurentia }\end{array}$ & $\begin{array}{l}\text { MC- } \\
\text { UC }\end{array}$ & $(S 116)$ \\
\hline Whipple Cave Formation; Nevada, USA; Laurentia & $\mathrm{UC}$ & $(S 117)$ \\
\hline Shingle Limestone; Nevada, USA; Laurentia & $\mathrm{UC}$ & $(S 118)$ \\
\hline $\begin{array}{l}\text { Maynardville Limestone, Conasauga Group; } \\
\text { Tennessee, USA; Laurentia }\end{array}$ & $\mathrm{UC}$ & $(S 119)$ \\
\hline Survey Peak Formation; Alberta, Canada; Laurentia & $\mathrm{UC}$ & $(S 120)$ \\
\hline Nolichuky Formation; Virginia, USA; Laurentia & $\mathrm{UC}$ & $(S 121)$ \\
\hline $\begin{array}{l}\text { Morgan Creek Limestone, Wilberns Formation; } \\
\text { Texas, USA; Laurentia }\end{array}$ & $\mathrm{UC}$ & $(S 122)$ \\
\hline Dotsero Formation; Colorado, USA; Laurentia & $\mathrm{UC}$ & $(S 123)$ \\
\hline $\begin{array}{l}\text { Snowy Range Formation (=Galatin Formation); } \\
\text { Wyoming-Montana, USA; Laurentia }\end{array}$ & $\mathrm{UC}$ & $(S 124)$ \\
\hline Deadwood Formation; Dacota, USA; Laurentia & $\mathrm{UC}$ & $(S 125)$ \\
\hline $\begin{array}{l}\text { Pilgrim Formation; Wyoming-Montana, USA; } \\
\text { Laurentia }\end{array}$ & $\mathrm{UC}$ & $(S 126) ;$ age $(S 127)$ \\
\hline Bison Creek Formation; Alberta, Canada; Laurentia & UC & $(S 128)$ \\
\hline Waterflow Formation; Alberta, Canada; Laurentia & $\mathrm{UC}$ & $(S 129)$ \\
\hline Nopah Formation; California, USA; Laurentia & $\mathrm{UC}$ & $(S 130)$ \\
\hline Conococheague Limestone; Maryland, USA; & UC & $(S 130)$ \\
\hline
\end{tabular}




\begin{tabular}{|c|c|c|}
\hline Laurentia & & \\
\hline $\begin{array}{l}\text { Machinchang Formation; Malaysia; Eastern } \\
\text { Gondwana }\end{array}$ & UC & $(S 131)$ \\
\hline $\begin{array}{l}\text { Fenshan Formation; Jilin, China; Eastern } \\
\text { Gondwana }\end{array}$ & $\mathrm{UC}$ & $(S 132)$ \\
\hline $\begin{array}{l}\text { Shabakty Formation; southern Kazakhstan; } \\
\text { Kazakhstania }\end{array}$ & $\mathrm{UC}$ & $(S 133)$ \\
\hline $\begin{array}{l}\text { Mort Member, Ninmaroo Formation; Queensland, } \\
\text { Australia; Eastern Gondwana }\end{array}$ & $\mathrm{UC}$ & $(S 134)$ \\
\hline $\begin{array}{l}\text { Arrinthrunga Formation; Northern Territory, } \\
\text { Australia; Eastern Gondwana }\end{array}$ & $\mathrm{UC}$ & $(S 135)$ \\
\hline $\begin{array}{l}\text { Chopko Formation; Krasnoyarsk Region, Russia; } \\
\text { Siberia }\end{array}$ & $\mathrm{UC}$ & $(S 136)$ \\
\hline Chukuka Formation; Yakutia, Russia; Siberia & $\mathrm{UC}$ & $\begin{array}{l}\text { cited as calcareous } \\
\text { breccia in }(S 110)\end{array}$ \\
\hline Eyra Formation; Yakutia, Russia; Siberia & $\mathrm{UC}$ & $\begin{array}{l}\text { cited as calcareous } \\
\text { breccia in }(S 110)\end{array}$ \\
\hline Diringde Reef Massif; Yakutia, Russia; Siberia & $\mathrm{UC}$ & $\begin{array}{l}\text { cited as calcareous } \\
\text { breccia in }(S 110)\end{array}$ \\
\hline $\begin{array}{l}\text { Orakta Formation; Krasnoyarsk Region, Russia; } \\
\text { Siberia }\end{array}$ & $\mathrm{UC}$ & $\begin{array}{l}\text { cited as calcareous } \\
\text { breccia in }(S 110)\end{array}$ \\
\hline $\begin{array}{l}\text { Kulyumbe Formation; Krasnoyarsk Region, Russia; } \\
\text { Siberia }\end{array}$ & $\mathrm{UC}$ & $\begin{array}{l}\text { cited as calcareous } \\
\text { breccia in }(S 110)\end{array}$ \\
\hline Oldondo Formation; Yakutia, Russia; Siberia & $\mathrm{LO}$ & $(S 110)$ \\
\hline Fillmore Formation; Utah, USA; Laurentia & LO & $(S 137)$ \\
\hline House Limestone; Nevada, USA; Laurentia & $\mathrm{LO}$ & $(S 117)$ \\
\hline $\begin{array}{l}\text { St George Group; Newfoundland, Canada; } \\
\text { Laurentia }\end{array}$ & $\mathrm{LO}$ & $(S 138)$ \\
\hline Wahwah Limestone; Utah, USA; Laurentia & $\mathrm{LO}$ & $(S 118)$ \\
\hline Volkhov Stage; Leningrad Region, Russia; Baltica & LO & $(S 139)$ \\
\hline $\begin{array}{l}\text { Dumugol Formation; South Korea; Eastern } \\
\text { Gondwana }\end{array}$ & LO & $(S 140)$ \\
\hline $\begin{array}{l}\text { Mungok Formation; South Korea; Eastern } \\
\text { Gondwana }\end{array}$ & $\mathrm{LO}$ & $(S 141)$ \\
\hline
\end{tabular}

S1. S. W. F. Grant, Am. J. Sci. 290-A, 261-294 (1990).

S2. A. B. Fedorov, A. Yu. Zhuravlev, in “Biomineralization 93”, Seventh International Symposium on Biomineralization, Monaco, Program Abstr., November 17-20, 1993, p. 98 (1993).

S3. A. Yu. Zhuravlev, J. A. Gámez Vintaned, E. Liñán, E., in XXIII Jornadas de la Sociedad Española de Paleontología, Caravaca de la Cruz, Spain, 3-6 de Octubre de 2007, Libro de Resúmenes, J. C. Braga, A. Checa, M. Company, Eds. (Inst. Geol. Miner. Esp. Univ. Granada, 2007), pp. 229-230.

S4. R. A. Wood, J. P. Grotzinger, J. A. D. Dickson, Science 296, 2383-2386 (2002). S5. J. P. Grotzinger, W. A. Watters, A. H. Knoll, Paleobiology 26, 334-359 (2000). S6. Z. Chen, S. Bengtson, C.-M. Zhou, H. Hua, Z. Yue, Lethaia doi: 10.1111/j.15023931.2007.00040.x (2007).

S7. S. Bengtson, Fossils and Strata 15, 5-9 (1983). 
S8. H. Szaniawski, in Palaeobiology of Conodonts, R. J. Aldridge, Ed. (Ellis Horwood, Chichester, 1987), pp. 35-47.

S9. V. V. Khomentovsky, G. A. Karlova, Stratigr. Geol. Correlation 13, 21-34 (2005).

S10. S. Conway Morris, M. Chen, Geol. Mag. 126, 615-632 (1989).

S11. A. Kouchinski, S. Bengtson, Acta Palaeontol. Pol. 47, 431-444 (2002).

S12. A. Kouchinsky, Alcheringa 24, 65-81 (2000).

S13. W.M. Feng, X.N. Mu, A.V. Kouchinsky, Lethaia 34, 305-309 (2001).

S14. B. Runnegar, Alcheringa 9, 245-257 (1985).

S15. B. Runnegar, in Origin, Evolution, and Modern Aspects of Biomineralization in

Plants and Animals, R. E. Crick, Ed. (Plenum, New York, 1989), pp. 75-94.

S16. S. Bengtson, S. Conway Morris, B. J. Cooper, P. A. Jell, B. Runnegar, Mem.

Assoc. Australas. Palaeontols 9, 1-364 (1990).

S17. A. Kouchinsky, Acta Palaeontol. Pol. 45, 119-150 (2000).

S18. W. Feng, W. Sun, Acta Palaeontol. Pol. 48, 21-30 (2003).

S19. D. Mehl, Bull. Inst. océanogr. Monaco n. special 14, 377-385 (1996).

S20. S. M. Porter, Palaios 19, 178-183 (2004).

S21. N. P. James, C. F. Klappa, J. Sediment. Petrol. 53, 1051-1096 (1983).

S22. A. Yu. Zhuravlev, in Biotic Recovery from Mass Extinction Events, M. B. Hart,

Ed. (Geological Society Special Publication, London, 102, 1996), pp. 79-96.

S23. S. Conway Morris, J. S. Crampton, B. Xiao, A. J. Chapman, Palaeontology 40, 167-189 (1997).

S24. M. Steiner, G. Li, Y. Qian, M. Zhu, B.-D. Erdtmann, Palaeogeogr.

Palaeoclimatol. Palaeoecol. 254, 67-99 (2007).

S25. S. Bengtson, S. Conway Morris, in Origin and Early Evolution of the Metazoa, J.

H. Lipps, P. W. Signor, Eds. (Plenum, New York, 1992), pp. 447-481.

S26. G. T. Ushatinskaya, Trans. Paleontol. Inst. Russ. Acad. Sci. 262, 1-89 (in Russian) (1995).

S27. C. B. Skovsted, L. E. Holmer, Acta Palaeontol. Pol. 48, 1-20 (2003).

S28. N. V. Grigorieva, in 26th International Geological Congress, Paleontological and Stratigraphic Reports of Soviet Geologists (Nauka, Moscow, 1980), pp. 49-55.

S29. N. V. Esakova, E. A. Zhegallo, Trans. Joint Russ.-Mongol. Paleontol. Exped. 46, 1-216 (in Russian) (1996).

S30. S. Conway Morris, M. Chen, Palaeontology 34, 357-397 (1991).

S31. S. Conway Morris, M. Chen, J. Paleontol. 66, 384-406 (1992).

S32. N. C. Hughes, G. O. Gunderson, M. J. Weedon, J. Paleontol. 74, 828-838 (2000).

S33. J. Pojeta, Jr., B. Runnegar, U. S. Geol. Surv. Prof. Pap. 968, 1-88 (1976).

S34. B. Runnegar, U. S. Geol. Surv. Prof. Pap. 968, 1-88 (1996).

S35. B. Runnegar, C. Bentley, J. Paleontol. 57, 73-92 (1983).

S36. V. Berg-Madsen V., Alcheringa 11, 245-249 (1987).

S37. A. V. Kouchinsky, Lethaia 32, 173-180 (1999).

S38. M. D. Brasier, R. M. Corfield, L. A. Derry, A. Yu. Rozanov, A. Yu. Zhuravlev, Geology 22, 455-458 (1994).

S39. P. D. Kruse, A. Yu. Zhuravlev, N. P. James, Palaios 10, 291-321 (1995).

S40. A. Yu. Rozanov, A. Yu. Zhuravlev, in Origin and Early Evolution of the Metazoa, J. H. Lipps, P. W. Signor, Eds. (Plenum, New York, 1992), pp. 205-282.

S41. E. Landing, G. Geyer, K. E. Bartowski, J. Paleontol. 76, 287-305 (2002).

S42. G. T. Ushatinskaya, A. Yu. Zhuravlev, Dok. Akad. nauk 337, 231-234 (in Russian) (1994). 
S43. A. Williams, C. H. C. Brunton, S. J. Carlson, Eds., Treatise on Invertebrate Paleontology, Pt H, Brachiopoda, Vol. 2 (Geological Society of America, Univ.

Kansas, Boulder, CO, 2000).

S44. W. C. Jones, in Biologie des spongiaires, Colloq. Int. CNRS, 291, 425-447 (1979).

S45. A. Yu. Zhuravlev, F. Debrenne, J. Lafuste, Cour. Forsch.-Inst. Senckenberg 164, 365-372 (1993).

S46. R. A. Wood, A. Yu. Zhuravlev, A. Chimed Tseren, Sedimentology 40, 829-858 (1993).

S47. M. Hicks, J. Paleontol. 80, 609-615 (2006).

S48. M. Fuller, R. Jenkins, Palaeontology 50, 961-980 (2007).

S49. C. B. Skovsted, Paläontol. Zeitschr. 77, 429-443 (2003).

S50. J. Lafuste, F. Debrenne, A. Zhuravlev, C. R. Acad. Sci. Paris, Ser. II 310, 15531559 (1990).

S51. N. V. Wilmot, A. E. Fallick, Palaeontology 32, 297-304 (1989).

S52. R. R. Gaines, M. L., Geology 31, 941-944 (2003).

S53. U. Brand, Chem. Geol. 204, 23-44 (2004).

S54. M. R. Lee, C. Torney, A. W. Owen, Palaeontology 50, 1031-1037 (2007).

S55. L. G. Voronova et al., Trans. Paleontol. Inst. USSR Acad. Sci. 224, 1-88 (in

Russian) (1987).

S56. S. E. Gabbott, D. J. Siveter, R. J. Aldridge, J. N. Theron, Lethaia 36, 151-160 (2003).

S57. C. D. Vann, T. M. Cronin, G. S. Dwyer, Mar. Micropaleontol. 53, 261-277 (2004).

S58. O. Tinn, T. Meidla, Palaeontology 47, 199-221 (2004).

S59. G. T. Ushatinskaya, Ya. E. Malakhovskaya, in The Evolution of Biosphere and Biodiversity, S. V. Rozhnov, Ed., (Tovarishchestvo nauchnykh izdaniy KMK, Moscow, 2006), pp. 177-192 (in Russian).

S60. J. A. D. Dickson, J. Sediment. Res. 74, 355-365 (2004).

S61. K. J. Müller, J. F. Miller, Lethaia 9, 391-395 (1976).

S62. R. Wrona, Palaeontol. Polon. 43, 9-16 (1982).

S63. S. E. Bendix-Almgren, J. S. Peel, Bull. Geol. Surv. Den. 37, 83-103 (1988).

S64. L. E. Holmer, C. B. Skovsted, A. Williams, Palaeontology 45, 875-882 (2002).

S65. G. C. O. Bischoff, Senckenb. Lethaea 69, 467-521 (1989).

S66. S. Bengtson, S. C. Mattews, V. V. Missarzhevsky, in Problematic Fossil Taxa, A. Hoffman, M. H. Nitecki, Eds. (Clarendon Press, Oxford), pp. 97-115 (1986).

S67. P. D. Taylor, M. A. Wilson, J. Paleontol. 73, 38-48 (1999).

S68. P. D. Taylor, M. J. Weedon, Zool. J. Linn. Soc. 128, 337-399 (2000).

S69. A. M. Smith, M.M. Key, Jr., D. P. Gordon, Earth-Sci. Rev. 78, 287-306 (2006).

S70. F.-S. Xiao, S.-G. Zhang, Z.-Z. Wang, J. Paleontol. 81, 1308-1326 (2007).

S71. C.-M. Yoo, Y.-I. Lee, Carbonates Evaporites, 8, 224-229 (1993).

S72. B. D. Webby, in The Great Ordovician Biodiversification Event, B. D. Webby, F.

Paris, M. L. Droser, I. G. Percival, Eds. (Columbia Univ. Press, New York, 2004), pp. 112-118.

S73. C. T. Scrutton, Proc. Yorks. Geol. Soc. 52, 1-57 (1998).

S74. B. D. Webby, R. J. Elias, G. A. Young, B. E. E. Neuman, D. Kaljo, in The Great Ordovician Biodiversification Event, B. D. Webby, F. Paris, M. L. Droser, I. G.

Percival, Eds. (Columbia Univ. Press, New York, 2004), pp. 124-146.

S75. R. E. Crick, Paleobiol. 7, 216-229 (1981).

S76. J.-y. Chen, D.-1. Qi, Acta Palaeontol. Sin. 21, 392-403 (in Chinese) (1982). 
S77. C. Teichert, in The Mollusca, Vol. 12: Paleontology and Neontology of

Cephalopods, M. R. Clarke, E. R. Trueman, Eds. (Academic, New York, 1988), pp. 1179.

S78. W. Haas, Biomineralization Res. Reps 5, 1-52 (1972).

S79. J. G. Carter, R. M. Hall, in Skeletal Biomineralization: Patterns, Processes and Evolutionary Trends, J. G. Carter, Ed. (Van Nostrand Reinhold, New York, 1990), v. 2, pp. 297-411.

S80. B. L. Stinchcomb, G. Darrough, J. Paleontol. 69, 52-65 (1995).

S81. M. E. Tucker, Sediment. Geol. 43, 67-84 (1985).

S82. M. E. Tucker, J. Geol. Soc. Lond. 149, 655-668 (1992).

S83. M. E. Tucker, Nature 319, 48-50 (1986).

S84. U. Singh, J. Sediment. Petrol. 57, 117-127 (1987).

S85. J. J. Álvaro, S. Clausen, A. El Albani, E. H. Chellai, Sedimentology 53, 35-53 (2006).

S86. A. I. Varlamov, V. M. Sundukov, Trans. Sib. Sci.-Res. Inst. Geol. Geophys. Miner.

Res. 270, 31-49 (in Russian) (1979).

S87. F. Debrenne. M. Debrenne, Beringeria Spec. Issue 2, 121-145 (1995).

S88. F. A. Corsetti, D. L. Kidder, P. J. Marenco, Sediment. Geol. 191, 135-150 (2006).

S89. W. Buggisch, G. F. Webers, Facies 7, 199-228 (1982).

S90. R. Wrona, A. Yu. Zhuravlev, Palaeontol. Pol. 55, 9-36 (1996).

S91. F. Debrenne, A. Gandin, G. L. Pillola, Riv. Ital. Paleontol. Stratigr. 94, 483-514 (1988).

S92. P. A. Sandberg, Nature 305, 19-22 (1983).

S93. J.-L. Mansy, F. Debrenne, A. Yu. Zhuravlev, Geobios 26, 643-683 (1993).

S94. J. F. Read, R. W. Pfeil, J. Sediment. Petrol. 53, 761-778 (1983).

S95. M. A. S. McMenamin, F. Debrenne, A. Yu. Zhuravlev, Geobios 33, 693-708 (2000).

S96. J. J. Álvaro, E. Vennin, D. Vizcaïno, Trans. R. Soc. Edinburgh: Earth Sci. 89 (for 1998), 135-143 (1999).

S97. O. Elicki, J. Schneider, R. Shinaq, Bull. Soc. géol. Fr. 173, 547-552 (2002).

S98. C. B. de Wet, J. A. D. Dickson, R. A. Wood, S. B. Gaswirth, H. M. Frey,

Sediment. Geol. 128, 13-21 (1999).

S99. N. Chow, N. P. James, J. Sediment. Petrol. 57, 907-921 (1987).

S100. M. V. Stepanova, in Paleoecological and Lithological-Facies Analyses for the Grounds of Detailed Regional Stratigraphic Schemes, V. I. Krasnov, Ed. (Sib. Sci.-Res. Inst. Geol. Geophys. Miner. Res., Novosibirsk, 1986), pp. 22-30 (in Russian).

S101. S. G. Whittaker, N. P. James, T. K. Kyser, Geochim. Cosmochim. Acta 58, 55675577 (1994).

S102. B. R. Pratt, Geol. Assoc. Can. Annu. Meeting 16, 102 (1991).

S103. G. M. Narbonne, S. M. Arbuckle, Can. Soc. Petrol. Geologists Mem. 13, 156-160 (1989).

S104. J. M. Kennard, N. Chow, N. P. James, Can. Soc. Petrol. Geologists Mem. 13, 151-155 (1989).

S105. B. Hamdi, A. Yu. Zhuravlev, P. D. Kruse, N. Y. State Mus. Bull. 492, 88 (1997).

S106. W. J. Johnson, R. H. Goldstein, Nature 362, 335-337 (1993).

S107. K. J. Tobin, S. M. Bergstrom, Palaeogeogr. Palaeoclimatol. Palaeoecol. 181, 399-417 (2002).

S108. J. F. Mount, D. Kidder, Sedimentology 40, 315-329 (1993).

S109. D. I. Gravestock et al., Trans. Palaeontol. Inst. Russ. Acad. Sci. 282, 1-344 (2001). 
S110. V. A. Astashkin et al., Internat. Union Geol. Sci. Publ. 27, 1-133 (1991).

S111. M. Keller, Argentine Precordillera: Sedimentary and Plate Tectonic History of a Laurentian Crustal Fragment in South America, Geol. Soc. Am. Spec. Pap. 341 (1999). S112. B. R. Pratt, O. L. Bordonaro, J. Sediment. Res. 77, 256-262 (2007).

S113. A. Pope, B. C. Min. Energy Mines Petrol. Res. Open File 1990-26 (1990).

S114. R. A. Robison, Geol. Soc. Am. Bull. 75, 995-1010 (1964).

S115. X. Meng, M. Ge, M. Tucker, Sediment. Geol. 114, 189-222 (1997).

S116. N. Chow, N. P. James, Am. Assoc. Pet. Geol. Bull. 68, 462 (1984).

S117. H. E. Cook, M. E. Taylor, Geology 3, 559-562 (1975).

S118. J. D. Cooper, Ed., Ordovician of the Great Basin: Fieldtrip Guidebook and

Volume for the Seventh International Symposium on the Ordovician System, Las Vegas, Nevada, USA, June 1995 (Pacific Section Society for Sedimentary Geology, Fullerton, CA) (1995).

S119. B. Glumac, K. R. Walker, Palaios 12, 98-110 (1997).

S120. Z. Ji, C. R. Barnes, J. Paleontol. 70, 871-890 (1996).

S121. J. R. Markello, J. F. Read, Sedimentology 28, 573-598 (1981).

S122. B. R. Spincer, J. Paleontol. 72, 577-584 (1998).

S123. P. Myrow et al., Geol. Soc. Am. Bull. 115, 695-713 (2003).

S124. P. M. Myrow et al., Sedimentology 511, 973-996 (2004).

S125. B. R. Pratt, Geology 30, 423-426 (2002).

S126. P. Kozub, in Proceedings of the Keck Research Symposium in Geology, C. V.

Mendelson, C. Manciewicz, Eds., 10, 134-137 (1997).

S127. M. R. Saltzman, J. Sediment. Res. 69, 926-938 (1999).

S128. S. R. Westrop, Lethaia 19, 123-132 (1986).

S129. B. B. Waters, Can. Soc. Petrol. Geologists Mem. 13, 165-169 (1989).

S130. R. S. Shapiro, S. M. Awramik, J. Paleontol. 80, 411-422 (2006).

S131. C. P. Lee, Palaeworld 15, 242-255 (2006).

S132. J.-Y. Chen et al., Geol. Mag. 125, 415-444 (1988).

S133. V. G. Zhemchuzhnikov, Lithol. polezn. iskop. 6, 76-87 (in Russian) (June 1986).

S134. E. C. Druce, J. H. Shergold, B. Radke, Natl Mus. Wales Geol. Ser. 3, 193-209 (1982).

S135. P. D. Kruse, L. C. Mohammed, J. N. Dunster, M. L. Duffett, Sandover River, Northern Teritory (Northern Territory Geological Survey, Darwin, 1:250000 Geol. map ser. explanatory notes SF 53-8, ed. 2, 2002).

S136. A. I. Varlamov, K. L. Pak, A. V. Rosova, Paleontol. J. 40 (suppl. 1), 1-56 (May 2006).

S137. B. F. Dattilo, Brigham Young Univ. Geol. Stud. 39, 71-100 (1993).

S138. B. R. Pratt, N. P. James, Sedimentology 33, 313-343 (1986).

S139. S. V. Rozhnov, in The Ecology of the Cambrian Radiation, A. Yu. Zhuravlev, R.

Riding, Eds. (Columbia Univ. Press, New York, 2001), pp. 238-253.

S140. Y. I. Lee, J. C. Kim, Sedimentology 39, 951-969 (1992).

S141. Y. S. Choi, J. C. Kim, Y. I. Lee, J. Geol. Soc. Korea 29, 15-29 (1993). 